ISBN 978-981-14-1684-2

Proceedings of 2019 the 9th International Workshop on Computer Science and Engineering

(WCSE 2019 SUMMER)

Hong Kong, 15-17 June, 2019, pp. 288-294

doi: $10.18178 /$ wcse. 2019.06 .043

\title{
Sentiment Analysis of LMS Users Using Support Vector Algorithm
}

\author{
Evelyn M. Baesa ${ }^{\#^{+}}$, Rosemarie T. Bigueras ${ }^{\#}$, Josephine Dela Cruz ${ }^{*}$, Daniel E. Maligat Jr. ${ }^{\#}$, Jocelyn \\ O. Torio $\#$, \\ \# ICS Department, Camarines Norte State College, Daet, Camarines Norte, 4600, Philippines \\ E-mail: vyneelbasea@gmail.com, rosemariebigueras@gmail.com,dmj234@yahoo.com, \\ jocelynt77@gmail.com \\ *University of the Cordilleras, Governor Park, Baguio City, 2600, Philippines \\ E-mail: delacruzpen@gmail.com
}

\begin{abstract}
This Higher education institutions are now being transformed by digital technologies in terms of administrative operations and delivery of instruction using learning management systems (LMS). This study aims to determine and understand the faculty and students' perceptions, experiences, expectations and concerns regarding educational uses of learning management systems (LMS). Qualitative research method was used to better understand the faculty and students' perceptions, expectations and concerns about LMS, responses from two (2) open-ended online survey questions was used as a primary source of data. For the statistical method which is also embedded in the processing technique, Support Vector Machine was used to validate the correctly classified instances. The study reveals that faculty and students exhibited positive perceptions in practicing and using learning management systems while the biggest expectations of faculty and students are assessment feature, discussion, accessibility, interface and hardware and software. The result of this study reveals that the perception achieved an average accuracy of $91.8182 \%$. Based on the findings of expectations and concerns, LMS must consider assessment feature, discussion, accessibility, interface and hardware and software to be an effective e- learning tools.
\end{abstract}

Keywords: Learning Management Systems; Sentiment Analysis; Support Vector Machine

\section{Introduction}

One of the most significant changes and challenges in education arena nowadays is the implementation of Information and Communication Technology (ICT) which provide new ways and capabilities to carry out learning processes [1]. ICT penetrates all human activities ranging from economic, business, banking, administration, education and communication since last two (2) decades [2]. In the field of education particularly in higher education, most universities and colleges utilize the ICT in administrative and academic areas including learning and delivery of instructional and educational resources by development of Institutional Learning Management System (ILMS).

This LMS allows coordination, delivery, and recovery of online course resources and materials, cater online communication and feedback between, faculty members and students [3]. Assessment will be given in the system to monitor students' performance by the instructors and students themselves. Top five (5) LMS features used by the instructors which includes placing syllabus or course materials, messaging or feedbacks, grade books, students submitting assignments and giving links to online resources [4]. These features are common in most of the LMS tools used by instructors and students since instructors in higher educational institutions conduct some instructional jobs often using a learning management system (LMS) tool than other instructional jobs [5].

Hence, this Learning Management System (LMS) have been proven to urge a useful way to deal with

+ Corresponding author. Tel.: +639175602726; +639496513187

E-mail address: vyneelbasea@gmail.com; delacruzpen@gmail.com 
information securing and bolster dynamic learning. One of the means to a fruitful and productive utilization of LMS is the means by which the partners receive and see this learning application. It is important to understand instructors' and students' perception of LMS in order to foresee some possible expectations and issues and help to fabricate a profitable learning condition and a submitted client network [6].

In numerous organizations, face to face instructing is exceptionally esteemed in spite of the fact that there are a few occasions where completely web-based learning is utilized for instructor training regardless of some instructors' apparent negligence taking part in compelling academic systems in online condition [7].

To be able to get the sentiments of faculty and students as well as to identify the main topic regarding the use of LMS tools in education, a machine learning is used to be more accurate in predicting outcome and is becoming a primary mechanism for extracting information [8]. In this study, opinion analysis through online responses, can help greatly from automatic topic mining which is provided by topic models like latent Dirichlet allocation (LDA) [9].

The purpose of this study is to determine the perceptions of students and faculty in using LMS tools, and determine the expectations and concerns of students and faculty in using LMS tools. The researchers used Support Vector Machine as classifier since this algorithm is suitable in the data of this research to find the best possible outputs in classification of data.

\section{Methodology}

The researchers used qualitative research method to collect and analyze data needed about the perception, expectation and concern in LMS. The questionnaire used was formulated and validated by a social science researcher which is composed of two (2) open-ended questions. These questions were given directly to get the sentiments of both students and faculty regarding the use of LMS as an educational tool. The data gathered by the researchers has a total of 1,321 responses for perception, concerns and expectations of the user regarding education uses of LMS.

The method structure of this study is composed of data collection, data cleaning, data annotation through manual classification of perception into positive or negative, and machine learning of the training set which is shown in figure 1 . The researchers manually classified the collected responses into negative perception or positive perception. To test the performance evaluation of the classification model, the researchers used 10 stratified cross-validation with a support vector algorithm that is applied in machine learning tools. One of today's most rapidly growing technical fields is machine learning that is placed at the juncture of computer science and statistics and at the central of data science where in this study, it is used to validate the performance of the data model then expectation and concern is classified through topic modelling [10].

In this study, sentiment analysis is done to label whether the sentiment is a positive or negative, Latent Dirichlet Allocation (LDA) is the algorithm used in topic modelling in generating the top topics amongst expectations and concerns of LMS users. Whereas, the results in topic modelling can be associated with the results in sentiments of faculty and students as a whole.

\subsection{Data collection and data source}

Survey questions was posted online through Google Docs/Sheets. Researchers gave the students and faculty the opportunity to respond to two (2) open-ended questions which are: What are your perceptions as in using LMS as tool in instructional delivery; and What are your expectations and concerns in using Learning Management System as tool in learning. Respondents' data and their responses was saved in spreadsheet application for data cleaning process.

\subsection{Data cleaning}

Since the data collected is noisy, the researchers undergo with the process of data cleaning in the form of removing duplicates, symbols, numbers and uppercase words. To remove duplicates, the machine learning tools were used is Waikato Environment for Knowledge Analysis (WEKA).

The researchers write code through writing regular expressions in Notepad++ to process symbols, uppercase and lowercase as another way of cleaning the data. After data cleaning only 770 responses on 
perception was retained.

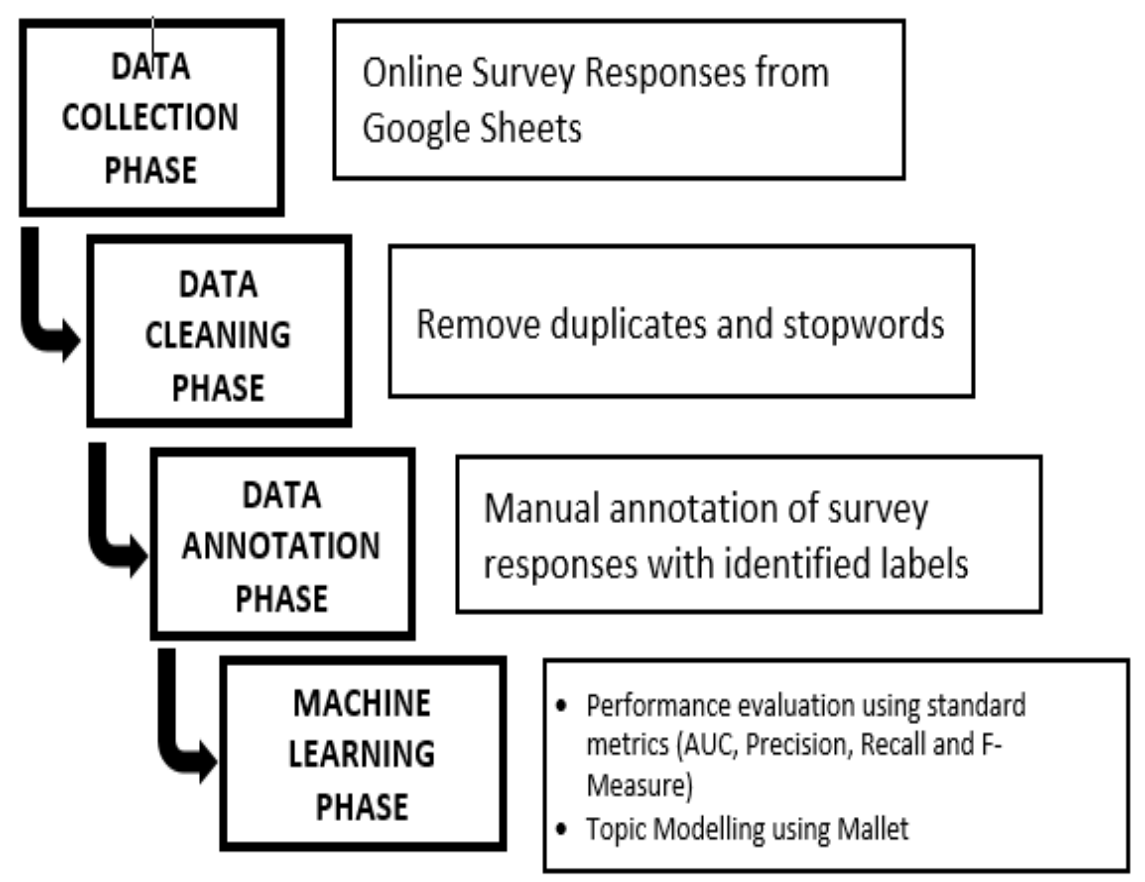

Figure. 1: Methodology Structure of the Study

\subsection{Annotation of data}

The researchers manually classified the collected perception responses data into positive or negative perceptions, while the expectations and concerns will be determined through topic modelling using mallet tool. Negative responses classified by researcher if the response has negative auxiliary words such as "do not", "not", etc. Table 1 show the example of cleaned classified positive and negative response:

Table 1: Sample Positive and Negative Responses

\begin{tabular}{|l|l|}
\hline Positive Response & Negative Response \\
\hline $\begin{array}{l}\text { helpful tool many topics discussed achieve depth } \\
\text { knowledge }\end{array}$ & not good instructional tool \\
\hline $\begin{array}{l}\text { allows seamless deliverance information students } \\
\text { guiding faculty }\end{array}$ & nobody explains lessons read only learn alone \\
\hline manageable easy & not useful \\
\hline another innovative way delivering lessons & not applicable mathematics engineering subjects \\
\hline user friendly easy manage accurate effective & not like cannot absorb lessons given online \\
\hline
\end{tabular}

\subsection{Machine learning tools and algorithms for classification}

For identifying and evaluating the manually classified and labelled perceptions of the researchers, the Waikato Environment for Knowledge Analysis (Weka) was used in this study as machine learning tools. In training machine learning tools for classifying positive and negative perceptions the supervised learning was used. Supervised learning means that the researchers already manually classified and labelled the perceptions into positive perceptions and negative perceptions to train the machine learning tools. To test the performance of the classification model of the perceptions, the test options used by the researchers is the stratified crossvalidation with ten (10) number of folds. The study titled Automatic Classification of Disaster-Related Tweet stated that the process of the machine learning tools in stratified cross-validation with ten (10) number of folds is the data set which is randomly split into 10 equal sizes of subsets, the manually classification model is trained is trained and tested 10 times, with the 9 -folds used as the training data set and the remaining 1-fold as the testing data set [11].

In this study, the algorithm Support Vector Machine (SVM) classifiers were used. This is the most 
commonly used machine learning algorithms for classification that is used for binary classification. Finding hyperplane that optimally separates the d-dimensional data into two classes is the main objective of SVM [12]. However, to make data more easily to separate, the SVM integrates the concepts of kernel that made feature space which makes the data into a higher dimensional space [13].

After classification and labelling of the perception responses, the researchers measure the reliability of classifying and labelling of perceptions using the Intraclass Correlation Coefficient (ICC) or multi-rater Kappa coefficient that is automatically computed by machine learning tools as it was used in this study. The intraclass correlation coefficient is the best measure for reliability for nonstop data [14]. The standard metrics such as accuracy, precision, recall and f- measure was used to test the training set performance evaluation is shown in Table 2.

Table 2: Metrics of Evaluation

\begin{tabular}{|l|l|}
\hline Metric & \multicolumn{1}{c|}{ Description } \\
\hline Accuracy & $\begin{array}{l}\text { The percentage of true positive (TP) and true negative (TN) perceptions that is correctly classified } \\
\text { manually by the researchers over the total number of perception responses. }\end{array}$ \\
\hline Recall & $\begin{array}{l}\text { The percentage of correctly classified as true positive perception over the number of true positive } \\
\text { perceptions and false negative (FN) perceptions. }\end{array}$ \\
\hline F-measure & The percentage of precision multiply by two over the sum of the precision and recall \\
\hline
\end{tabular}

Legend:

True Positive -means that the manually labelled positive perceptions of the researcher is also classified by the machine learning tools as positive perceptions.

False Positive -means that the manually labelled positive perceptions of the researcher classified by the machine learning tools as negative perceptions. True Positive -means that the manually labelled positive perceptions of the researcher is also classified by the machine learning tools as positive perceptions.

False Positive - means that the manually labelled positive perceptions of the researcher classified by the machine learning tools as negative perceptions.

\section{Results and Discussion}

\subsection{Perceptions of students and faculty in using LMS tools}

The respondents of this study are the students and faculty members of higher education institutions in the Philippines who have a background or have used the LMS as a tool for learning. To classify positive or negative perception responses, the pre-processing method applied in this study is the cleaning of data through removing duplicate responses, symbols, numbers and uppercase words.

To test the performance evaluation of the classification model the researchers used 10 stratified cross validation with a support vector algorithm that is applied in machine learning tools. Figure 2 showed the summary results of the 10-folds stratified cross validation with the used of SVM as classifier algorithm of machine learning tools for checking the accuracy of the classifier model of perception responses.

Based on the summary results of stratified cross-validation of perception the SVM model attained an average accuracy of $91.8182 \%$, this means that the SVM model identified that 707 unique responses are correctly classified out of 770 unique responses. In terms of kappa statistics, .8257 is the result which means that the SVM classifier agreed on the classified instances by the researchers. In terms of recall, the SVM classifier classified 451 positive perceptions and only 10 positive responses classified as negative perceptions resulting to a recall value of $97.8 .2 \%$ for positive class, while for negative class the SVM classifier classified 256 negative perceptions and only 53 negative perception was classified as positive perceptions resulting to a recall value of $82.80 \%$ for a negative class. In terms of Precision metric, the perception was classified as $92.20 \%$ out of all the responses.

The SVM algorithm classified as positive and F-Measure shows that the classifications of the manual responses is correctly classified with a weighted mean of $91.70 \%$. The high percentage rating obtained on the True Positive (TP) of the binary classified data sets implied that the manually annotated data sets for 
perceptions are correct instances.

The perception of the students and faculty in using LMS is positive since LMS can enhance the traditional teaching and learning process of the users. LMS is also easy and manageable to use as well as it saves money and time.

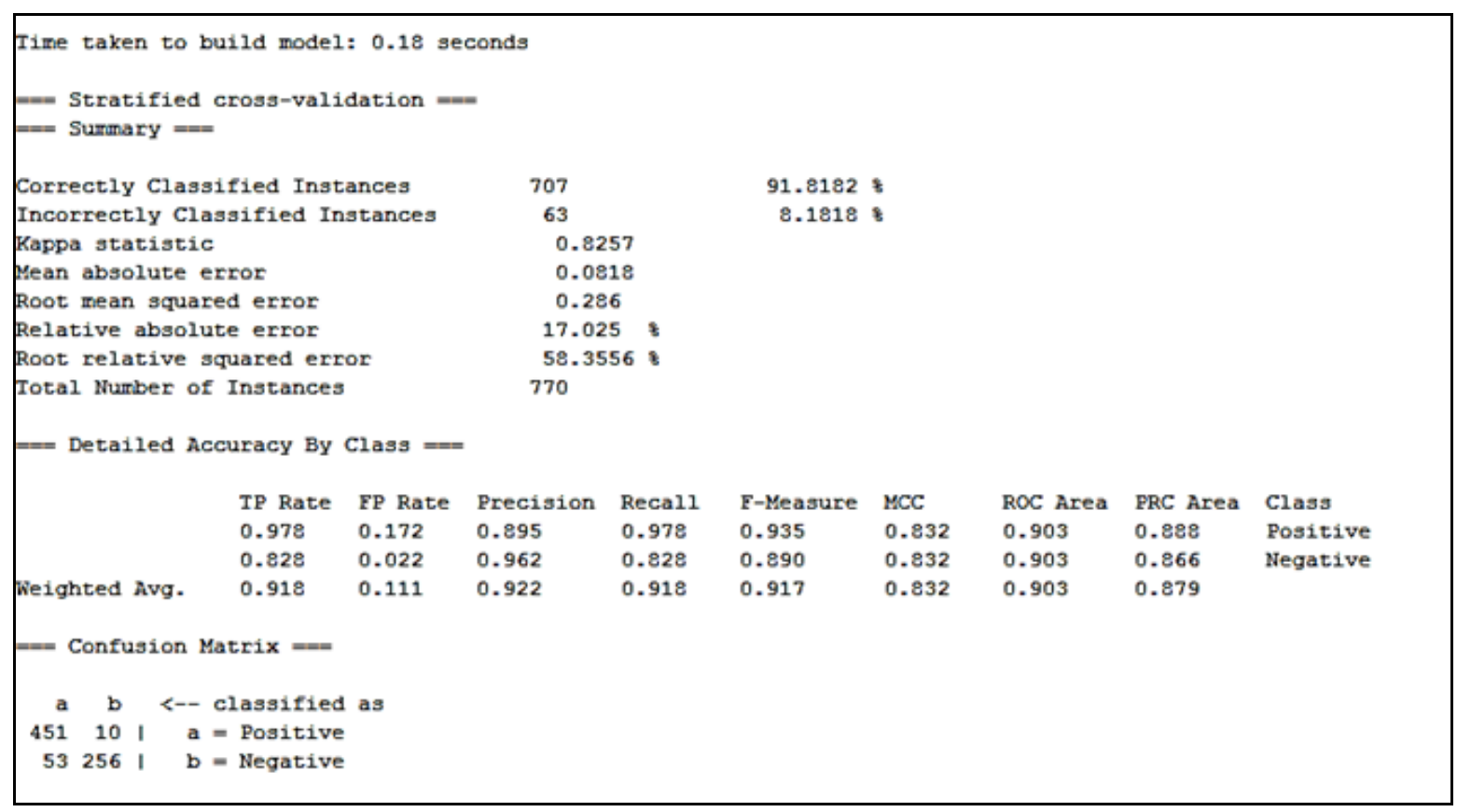

Figure. 2 Stratified Cross-Validation Summary Study

\subsection{Concerns and expectation on LMS}

Each topic model was identified based on the number of occurrences of unique word and the relationship or connection of each word with same meaning. In mallet tool, the Dirichlet parameter indicative of the weight of each generated topic model to obtained the weight of the topic labels. With the 770 data sets, a number of topics by 10 unique words were generated.

To identify the concerns and expectation of the users as learning tools, the researchers used topic modelling to classify the concerns and expectations of the users and labelled each. The following topic models were generated as shown in Table 3. Through the Dirichlet parameter indicative of the weight of each topic, assessment feature has the highest weight of $7.35 \%$ which indicates as the principal topic in the model.

Table 3. Topic Model Results

\begin{tabular}{|l|l|l|l|}
\hline No. & Weight & \multicolumn{1}{|c|}{$\begin{array}{c}\text { Topic } \\
\text { Labels }\end{array}$} & \multicolumn{1}{c|}{ Topic Models } \\
\hline 0 & 0.07358 & $\begin{array}{l}\text { Assessment } \\
\text { Feature }\end{array}$ & $\begin{array}{l}\text { quiz, checking, essay, evaluation, } \\
\text { exam, check, } \\
\text { assessment, gamification, } \\
\text { games, security }\end{array}$ \\
\hline 1 & 0.06966 & Discussion & $\begin{array}{l}\text { materials, interactive, face, bored, } \\
\text { self, teacher, students, } \\
\text { feedback, discuss, module }\end{array}$ \\
\hline 2 & 0.03701 & Accessibility & $\begin{array}{l}\text { Internet, connectivity, offline, easy, } \\
\text { free, accessibility, time, connection, } \\
\text { access, mobile }\end{array}$ \\
\hline 3 & 0.01755 & Interface & $\begin{array}{l}\text { design, friendly, dashboard, video, } \\
\text { enhanced, appearance, } \\
\text { graphics, color, text, layout }\end{array}$ \\
\hline
\end{tabular}




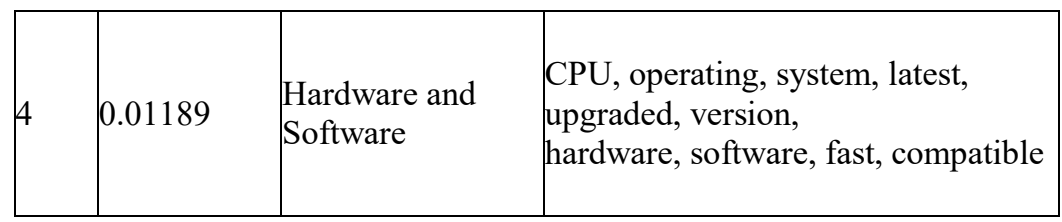

In this study, the researchers come up with the topic labels according to the top 10 topic models generated after running in mallet application. According to Schaffhauser (2015), an LMS program nowadays has the course-management basics which provides means for student to comply with the assignments given and for faculty members to conduct an online lectures or discussions and carry some announcements. Users nowadays are looking for features such as assessment features and social interface [15]. Assessment is one of the important feature since it will identify the level of learning of the users of LMS, it will also identify if the objective of the LMS was attained by the user.

Moreover, accessibility feature is also important since it will help the user to easily use the LMS which means it makes the user easily understand the direction or guide in the LMS. Furthermore, it is stated in the study of Kasim and Khalid (2016), that accessibility should also be considered as one of the characteristics of an LMS [16]. It is stated in the study of Mtebe \& Kondoro (2016) that the continued penetrating and embracing mobile phones in most of the population will produce to a more accessible LMS via mobile phones [17].

Hardware and software has a great impact in the efficiency of every information system or application for it is the main component of every application. Thus, hardware and software should also be in latest and upgraded version to run the application smoothly. Likewise, the interface of the application should also be considered in the development of LMS to be able to attract learners and to gain the interests of users as well.

Findings revealed that the concerns and expectations of the users in the LMS are the assessment, discussion, accessibility, interface, and hardware and software. Indication on the weight of each Dirichlet parameter showed that the topics identified were assessment (7.35\%), discussion (6.96\%), accessibility $(3.70 \%)$, interface $(1.75 \%)$, and hardware and software $(1.18 \%)$.

Based on the findings, the researchers analyzed that the LMS should be mostly improved in the assessment feature that is applied in different subjects, course and different type of exam as well as the checking tools of the LMS. Discussion feature should include interactive and availability of feedback for student and faculty. It also shows that fast and strong internet accessibly is also one of the concerns and expectations of faculty and students. LMS Interface, hardware and software are characteristics of LMS that users see equally important among others.

\section{Conclusions}

The main purpose of the study is to conduct sentiment analysis on the perceptions learning management system of students and faculty. It also aims to identify expectations and concerns about learning management system of student and faculty. This was made possible through the used 10 stratified cross validation with a support vector algorithm that is applied in machine learning tools

Topic modelling using mallet was also applied in this study to classify the different expectation and concerns of faculty and students using learning management system as learning tool.

The study reveals that faculty and students exhibited positive perceptions in practicing and using learning management systems while the biggest expectations of faculty and students are assessment feature, discussion, accessibility, interface and hardware and software.

The result of this study reveals a remarkable perception achieved an average accuracy of $91.8182 \%$. Based on the findings of expectations and concerns, LMS must consider the following, assessment feature, discussion, accessibility, interface and hardware and software to be an effective e- learning tools.

\section{Acknowledgment}

The researchers would like to thank Camarines Norte State College for supporting this study and the respondents for the responses and cooperation during data gathering. Support of the researcher's family is 
highly appreciated. Above all, the Almighty God for the knowledge, wisdom and strength to pursue this study.

\section{References}

[1] Conde, M. Á., García-Peñalvo, F. J., Rodríguez-Conde, M. J., Alier, M., Casany, M. J., \& Piguillem, J. (2014). An evolving Learning Management System for new educational environments using 2.0 tools. Interactive Learning Environments, 22(2), 188-204. https://doi.org/10.1016/j.chb.2013.05.023

[2] Abdullah, H., Wahidin, I. S., Ibrahim, K., Rahman, S. A., Arshad, S. R., \& Zain, S. A. M. (2017). Awareness and perception among academicians in utilizing personalised learning management system: A case study. In 29th International Business Information Management Association Conference-Education Excellence and Innovation Management through Vision 2020: From Regional Development Sustainability to Global Economic Growth, IBIMA.

[3] Teasley, S. D., \& Lonn, S. (2007, July). Using learning management systems to support students' collaborative learning in higher education. In Proceedings of the 8th international conference on Computer supported collaborative learning (pp. 718-720). International Society of the Learning Sciences.

[4] Gomez, J. F. (2015). Higher education faculty use of a learning management system in face-to-face classes (Doctoral dissertation).

[5] Shin, W. S., \& Kang, M. (2015). The use of a mobile learning management system at an online university and its effect on learning satisfaction and achievement. The International Review of Research in Open and Distributed Learning, 16(3).

[6] Emelyanova, N., \& Voronina, E. (2014). Introducing a learning management system at a Russian university: Students' and teachers' perceptions. The International Review of Research in Open and Distributed Learning, 15(1). http://dx.doi.org/10.19173/irrodl.v15i1.1701

[7] Downing, J. J., \& Dyment, J. E. (2013). Teacher Educators' Readiness, Preparation, and Perceptions of Preparing Preservice Teachers in a Fully Online Environment: An Exploratory Study. The teacher educator, 48(2), 96-109. https://doi.org/10.1080/08878730.2012.760023

[8] Xing, E. P., Ho, Q., Dai, W., Kim, J. K., Wei, J., Lee, S., ... \& Yu, Y. (2015). Petuum: A new platform for distributed machine learning on big data. IEEE Transactions on Big Data, 1(2), 49

[9] Nikolenko, S. I., Koltcov, S., \& Koltsova, O. (2017). Topic modelling for qualitative studies. Journal of Information Science, 43(1), 88-102

[10] Jordan, M. I., \& Mitchell, T. M. (2015). Machine learning: Trends, perspectives, and prospects. Science, 349(6245), 255-260.

[11] Parilla-Ferrer, B.E, Fernandez PL, Ballena JT. Automatic Classification of Disaster-Related Tweets. InProc. International conference on Innovative Engineering Technologies (ICIET) 2014 Dec (p. 62).

[12] Kumar, S., Gao, X., Welch, I., \& Mansoori, M. (2016, March). A machine learning based web spam filtering approach. In Advanced Information Networking and Applications (AINA), 2016 IEEE 30th International Conference on (pp. 973-980). IEEE.

[13] Li, W., Prasad, S., \& Fowler, J. E. (2014). Decision fusion in kernel- induced spaces for hyperspectral image classification. IEEE Transactions on Geoscience and Remote sensing, 52(6), 3399-3411.

[14] Zafarani, R., Abbasi, M., Liu, H. (2014). Social Media Mining: An Introduction. Cambridge University Press, 2014.

[15] Schaffhauser, D. (2015). 4 Features to Look for in a 21st Century LMS: Two Districts Share Their Experiences of Choosing a Learning Management System That Does a Lot More Than Help Teachers Post Assignments. THE Journal (Technological Horizons In Education), 42(4)

[16] Kasim, N. N. M., \& Khalid, F. (2016). Choosing the right learning management system (LMS) for the higher education institution context: a systematic review. International Journal of Emerging Technologies in Learning (iJET), 11(06), 55-61.

[17] Mtebe, J. S., \& Kondoro, A. W. (2016, May). Using Mobile Moodle to enhance Moodle LMS accessibility and usage at the University of Dar es Salaam. In IST-Africa Week Conference, 2016 (pp. 1-11). IEEE. 